\title{
SOME OBSERVATIONS ON THE MASKING EFFECTS OF TWO-DIMENSIONAL STIMULI
}

\author{
A. M. Derrington and G. Bruce Henning ${ }^{1 *}$ \\ The Department of Physiological Sciences, The Medical School, Framlington Place, Newcastle-upon- \\ Tyne, NE2 4HH, U.K. and 'David Sarnoff Research Center†, P.O. Box 432, Princeton, NJ 08540, U.S.A.
}

(Received 23 November 1987; in revised form 3 May 1988)

\begin{abstract}
Gratings that differ in orientation by as much as $62.5 \mathrm{deg}$ from that of a signal grating raise the signal's threshold by nearly a $\log$ unit. The spatial-frequency tuning of the masking effect reaches a maximum slightly below the spatial frequency of the maskers but far from that of any quadratic distortion product. Further, the location of the peak does not depend much on the relative orientation of the signal and maskers thus making it unlikely that the masking effect can be explained in any simple way by the presence of visual nonlinearities. This illustrates the difficulty of attempting to explain human performance in even relatively simple discrimination experiments with models based on mechanisms tuned for spatial frequency and orientation.
\end{abstract}

\section{INTRODUCTION}

There is a variety of evidence consistent with the view that visual patterns, at least in the relatively peripheral parts of the visual system, are processed by arrays of visual mechanisms (channels) each of which responds to stimuli over only a limited range of spatial frequency and orientation (Campbell and Robson, 1968). Some of the properties of channels have been inferred from adaptation studies (Gelinski, 1968; Pantle and Sekuler, 1968; Blakemore and Campbell, 1969) and from studies of masking with one-dimensional noise (Greis and Roehler, 1970; Stromeyer and Julesz, 1972; Henning et al., 1981; Henning, 1988). Channels have been compared with simple cells in mammalian visual cortex which also behave as if they were summing devices sensitive to limited ranges of orientation and spatial frequency (Maffei and Fiorentini, 1973; De Valois et al., 1982).

One psychophysical observation that is difficult to reconcile with this view of the visual system is the finding that a contrast-modulated grating of high spatial-frequency which contains 3 components with spatial frequencies that are factors of $n-1, n$ and $n+1$ times their fundamental frequency makes gratings two octaves lower in spatial frequency difficult to see (Henning et al., 1975; Nachmias and Rogowitz,

\footnotetext{
"On leave from: The University of Oxford, Department of Experimental Psychology, South Parks Rd, Oxford OX1 3UD, U.K.
}

TA subsidiary of SRI International.
1983). This observation makes untenable the notion that the detection of such patterns is determined in any straight-forward way by linear, independent spatial-frequency selective mechanisms (channels); the contrast-modulated grating contains no components with spatial frequencies near the frequency of the signal and so should not affect its detectability.

It has been suggested that some aspects of masking by complex gratings are consistent with a simple second-order nonlinearity at a stage preceding the channel formation (Henning et al., 1975; Nachmias and Rogowitz, 1983). One effect of such a nonlinearity in response to a contrast modulated grating would be to introduce a component not present in the stimulusa "distortion product"-at the modulation frequency. Further, such distortion products have been observed in the responses of $X$-cells in the cat's lateral geniculate nucleus (LGN) (Derrington, 1987a). And it has been shown that the distortion product could cause contrast modulated gratings to mask the physiological response to gratings of the modulation frequency (Derrington, 1987b). It has also been argued that the even order of the nonlinearity that generates the distortion product allows it to be removed by relatively simple cortical processing, so that effectively linear higherlevel channels could be fabricated (Derrington, 1987a).

The aim of the experiments described here is to extend the psychophysical study of the hypothetical nonlinear mechanism by examining the masking effect of a stimulus comprising two 
gratings of the same spatial frequency but different orientation. Since the early levels of the visual pathway show little selectivity for stimulus orientation, an early nonlinearity should generate inter-modulation distortion products from generating gratings of different orientations on the retina. The distortion products will appear at known frequencies and orientations related to the frequencies and orientations of the generating gratings; where the generating gratings have the same spatial frequency and contrast (the case we consider) the distortion product is orientated half-way between the generating stimuli (see equation 7).

In the first experiment, we show that the sum of a pair of crossed gratings produces a substantial elevation of the threshold for detecting a grating with the same orientation as the expected distortion product. In subsequent experiments we explore the range of spatial frequencies and orientations producing masking. The results allow us to reject the hypothesis that the masking is produced by nonlinear distortion products that occur prior to orientation tuning.

\section{GENERAL METHODS}

There were two observation intervals on each trial and a masker consisting of either one or two sinusoidal gratings always appeared in both. The signal, a vertical sinusoidal grating, was presented in one of the observation intervals and the observers were required to indicate the interval in which it had occurred. The signal was in the first observation interval with probability 0.5 on each trial and, after each trial, the Observers were informed which interval had contained the signal.

Stimuli comprising three sinusoidal components were generated on the screen of a Hewlett-Packard 1317B $X-Y$ display (P/31 phosphor) using the method of Schade (1956). Lists of values (12-bits) describing the crosssectional luminance profiles of each field were linearized and stored as 256-element arrays in a computer (Hewlett-Packard 9836). The arrays were subsequently read out at a field rate of $200 \mathrm{~Hz}$ through 12-bit DACs to the display. Each component of the stimulus was presented in a separate field of the 3-field frame. Uniform fields replaced the signal grating in those observation intervals when no signal was presented and a uniform field also replaced components of the masker when they were not required.

The display was viewed binocularly and sub- tended a disk of 7 deg diameter at the observers' eyes; the surround was black and no spatial vignetting was used. The compound pattern was displayed for a nominal duration of $2 \mathrm{sec}$ but was shaped by a raised-cosine (Hanning) temporal window (Rabiner and Gold, 1975) which modulated the contrast of the stimuli. Each component of the masker had a contrast of approx. $20 \%$ and the presentation of the stimuli did not affect the mean luminance of the display $\left(13.2 \mathrm{~cd} / \mathrm{m}^{2}\right)$

To determine the appropriate contrast range of the psychometric function, trials were run initially in blocks of 10 . On successive blocks, the contrast of the signal was reduced by a factor of about $0.2 \mathrm{log}$ units. For contrasts close to the contrast corresponding to $75 \%$ correct responses, 90 more observations were made in two additional blocks of 40 and 50 trials each to make a total of 100 trials for one point above and one below $75 \%$ correct; the contrast corresponding to $75 \%$ correct was then estimated by linear interpolation.

\section{EXPERIMENT I: MASKING}

In this experiment, we measured the detectability of a 3-c/deg vertically orientated grating presented: (a) with no masker, i.e. on a uniform field; (b) with a single 3-c/deg diagonal masker orientated $+45 \mathrm{deg}$ from vertical; and (c) with two maskers orientated symmetrically $\pm 45 \mathrm{deg}$ from vertical. The components of the masker were generated with the same spatial frequency and phase as the signal.

\section{Results}

The psychometric functions relating the percentage of correct responses to the logarithm of signal contrast were approximately parallel. Thus discrimination performance can be represented legitimately by the signal contrast corresponding to a fixed level of correct responses, the "threshold"; we use the $75 \%$ level. Results for three Observers detecting a 3-c/deg vertical grating against maskers $45 \mathrm{deg}$ different in orientation are shown in the columns of Table 1: the first row gives the unmasked threshold, the next two rows give the masked thresholds for two- and one-component maskers, respectively. The bottom row gives the two-component threshold elevation-the ratio of the twocomponent threshold to the unmasked threshold. The masked threshold is, on average, about a factor of $9(0.95 \log$ units) above the un- 
Table 1. The contrast corresponding to $75 \%$ correct responses for three observers detecting a 3-c/deg vertical grating. The three rows show the results with no masker, with a pair of 3-c/deg maskers \pm 45 deg from vertical, and with a single masker $45 \mathrm{deg}$ from vertical, respectively

\begin{tabular}{llll}
\hline & \multicolumn{1}{c}{ PS } & \multicolumn{1}{c}{ AMD } & GBH \\
\hline No masker & 0.00275 & 0.00255 & 0.0024 \\
Two-comp. & 0.0210 & 0.0251 & 0.0211 \\
$\begin{array}{l}\text { One-comp. } \\
\text { Threshold elevation }\end{array}$ & 0.00630 & 0.00604 & 0.00466 \\
factor & 7.6 & 9.8 & 8.8 \\
\hline
\end{tabular}

masked threshold. The elevation produced by a single masking component is much less, a factor of about 2.2 .

The large masking effect produced by masking components $45 \mathrm{deg}$ from the signal orientation is difficult to reconcile with the notion of signal detection through mechanisms tuned to narrow ranges of orientation.

\section{EXPERIMENT II: ORIENTATION AND SPATIAL-FREQUENCY TUNING}

To explore the effects of orientation and spatial-frequency tuning, we next measured the masking effect of two-component maskers orientated $\pm 22, \pm 45$ and $\pm 67.5 \mathrm{deg}$ either side of the vertical signal. The components of the masker had a spatial frequency of $3 \mathrm{c} / \mathrm{deg}$ in the direction normal to their orientation and the signal took a range of spatial frequencies above and below that of the maskers.

\section{Results}

Figures 1 and 2 show the results for two observers. In both figures detection performance with no masker is indicated by the solid line; the signal contrast corresponding to $75 \%$ correct responses is plotted on the abscissa as a function of spatial frequency and both axes are logarithmic. The reciprocal of the contrasts indicated by the solid line is the contrast sensitivity function obtained in our experimental conditions and reveals nothing unexpected; with the 7 deg circular field, sensitivity reaches a peak near $3 \mathrm{c} /$ deg.

The two-component masking results are shown by solid symbols: threshold signal contrast for masker orientation of $\pm 22.5 \mathrm{deg}$, triangles, \pm 45 deg circles, and \pm 67.5 deg squares. We were interested in the elevation produced by the maskers; that is, in the ratio of the masked to the unmasked thresholds.

These ratios are shown in Figs 3 and 4 where ..le logarithm of the threshold elevation

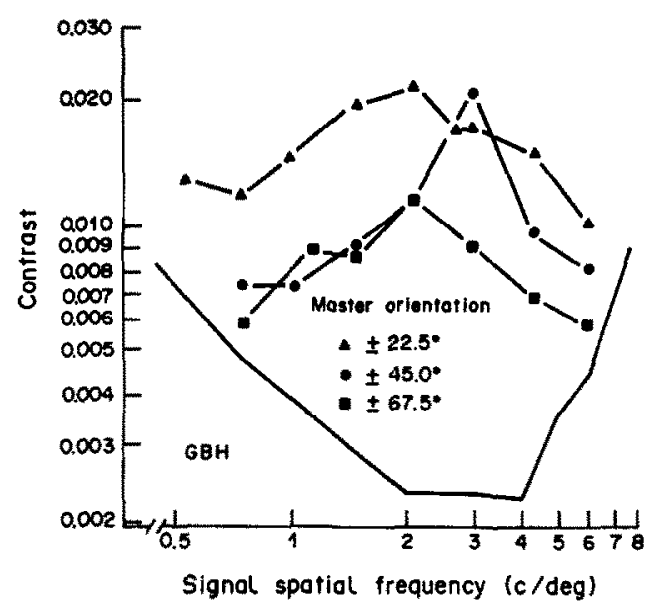

Fig. 1. The signal contrast for $75 \%$ correct detection as a function of spatial frequency. The solid line shows the results when the vertical signal is presented against a uniform background; the symbols show the result with 3 -c/deg maskers orientated: (a) $\pm 22.5 \mathrm{deg}$ from vertical (triangles); (b) $\pm 45 \mathrm{deg}$ from vertical (circles), and (c) $+67.5 \mathrm{deg}$ from vertical (squares). Data for Observer GBH.

is shown as a function of the spatial frequency of the signal. All orientations show broad spatial-frequency tuning with a peak slightly below the spatial frequency of the maskers. The tuning is in all cases very much broader than that revealed by sinusoidal maskers having the same orientation as the signal (Quick and Reichert, 1975; Henning and Hinton, unpublished data) and has the asymmetry found in noise masking experiments (Henning et al., 1981; Henning, 1988). It should be remembered in our experiment that the spatial frequency of the signal varies and the spatial frequency of the masker is held constant whereas in noise masking experiments, the signal frequency is held constant and the spatial frequency of the

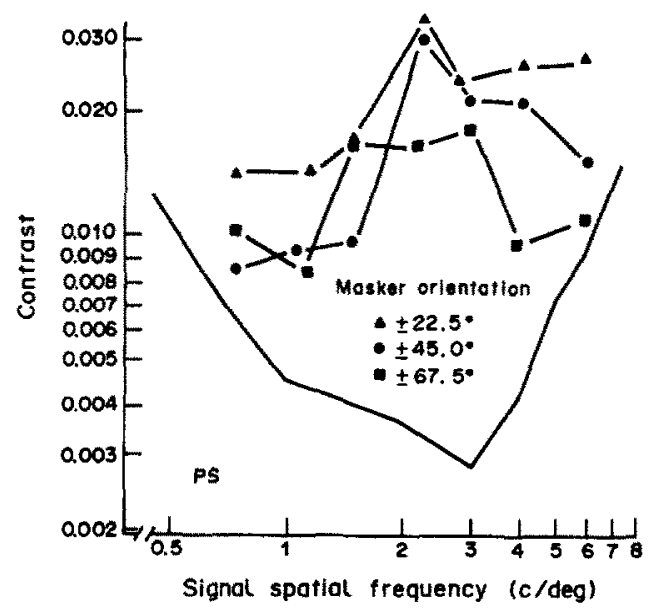

Fig. 2. Same as Fig. 1. Data for observer PS. 
masker varies; the procedural difference results in a reflexion of the masking function.

The vertical arrows in each graph indicate the spatial frequency of second-order distortion products the existence of which, we thought, might produce the masking we measure.

\section{DISCUSSION}

Philips and Wilson (1984) showed interaction between gratings of different orientation and found tuning widths of about $\pm 20 \mathrm{deg}$, with a monotonic decrease in interaction as the orientation of the masker deviated from that of the signal. In this context, the vertically tuned channel most sensitive to our signal might be expected to show some response to (and hence some masking from) the $22.5 \mathrm{deg}$ maskers. The 45 deg maskers should produce very little masking and the $67.5 \mathrm{deg}$ maskers virtually none. Figures 3 and 4 shows that this is not the case; there is almost as much masking with $67.5 \mathrm{deg}$ orientations as with $22.5 \mathrm{deg}$. The notion of linear orientation-tuned channels, like the notion of linear spatial-frequency tuned channels, cannot be used in any simple fashion to predict our results.

This is not the first demonstration that masking occurs between stimuli of very different orientation. Burbeck and Kelly (1981) showed that flickering horizontal gratings elevate the contrast for detecting flickering vertical gratings. Ferrara and Wilson (1987) showed that moving crossed gratings elevate the threshold for detecting moving patches of grating. Our results extend these findings by showing that

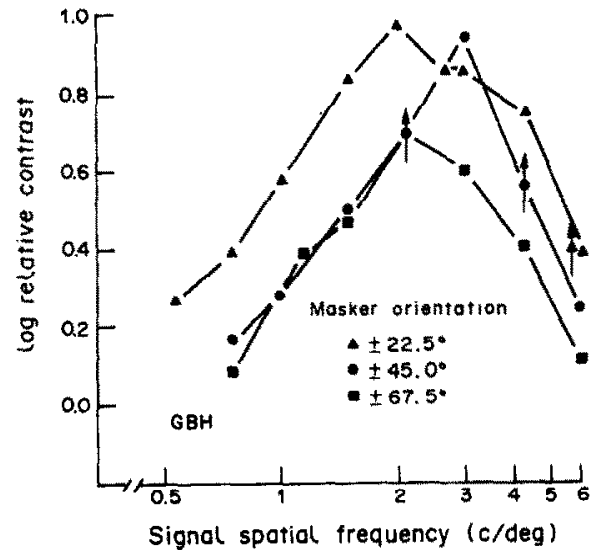

Fig. 3. Threshold elevation (the logarithm of the ratio of the masked to the unmasked thresholds) as a function of the vertical signal's spatial frequency. The arrows mark the spatial frequency of the vertical quadratic distortion prod uct. Data from observer GBH

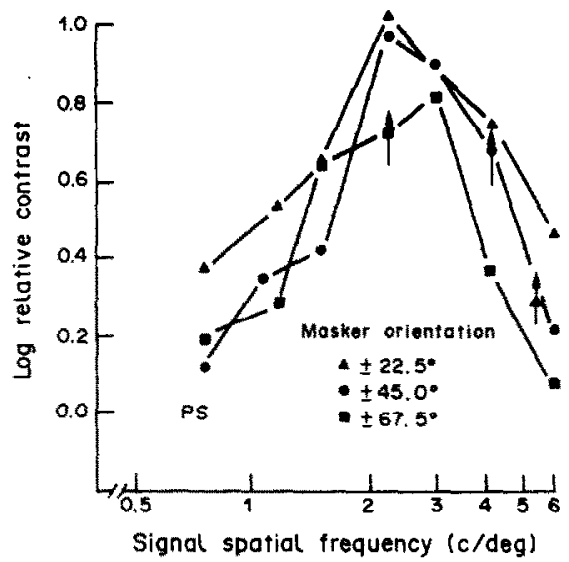

Fig. 4. Same as Fig. 3. Data for observer PS.

substantial interactions among gratings of different orientation occur at spatial and temporal frequencies generally associated with pattern vision rather than with the detection of motion or flicker. We thus demonstrate that interactions like those described by Ferrara and Wilson in the motion detection system also occur in pattern vision.

Another line of attack on the concept of simple linear orientation-tuned channels came on the basis of findings with gross evoked potentials (Regan and Regan, 1987); primary or generating gratings of different spatial frequency orientated at right angles produce evoked potential components that are not present in the responses to the gratings alone. These components have sometimes been attributed to nonlinearities and we shall now consider one explanation of our results based on nonlinear effects.

The two-dimensional luminance distribution of our masker, $L_{m}(x, y)$, is given by,

$$
\begin{aligned}
L_{m}(x, y)=L\left\{1+c\left(\sin \left[2 \pi\left(u_{1} x+v_{1} y\right)\right]\right.\right. \\
\left.\left.+\sin \left[2 \pi\left(u_{2} x+v_{2} y\right)\right]\right)\right\},
\end{aligned}
$$

where $\bar{L}$ is the mean luminance of the stimulus and $c$ is the contrast of each component of the masker. The parameters $u_{i}$ and $v_{i}$ determine the spatial frequency and orientation of the $i$ th component of the masker; the spatial frequency, $M F_{i}$, and orientation, $M \theta_{i}$ are simply,

$$
M F_{i}=\left(u_{i}^{2}+v_{i}^{2}\right)^{1 / 2},
$$

and

$$
M \theta_{i}=\tan ^{-1}\left(u_{i} / v_{i}\right),
$$

where, for convenience, $M \theta_{i}$ is measured relative to vertical. 
Because some care has been taken to ensure that it is so, equation 1 describes the stimulus presented to the observers' eyes. However, any nonlinearities in the transformations from retinal illuminance to the decision axes involved in our discrimination task may drastically alter the effective stimulus. One simple nonlinearity that has been considered extensively (Carter and Henning, 1971; Legge and Foley, 1980; Carlson and Klopfenstein, 1985; Regan and Regan, 1987; Derrington, 1987a) lies in the transformation of luminance to "effective" luminance. It is convenient to treat this nonlinearity as a power series and to consider only a few of the lower order terms of that expansion. For example, from the luminance distribution of equation 1 , the quadratic term in the series produces a distortion product, $D_{2}(x, y)$, given by,

$$
\begin{aligned}
D_{2}(x, y)= & 2 k_{2} c^{2}\left\{\sin \left[2 \pi\left(u_{1} x+v_{1} y\right)\right]\right. \\
& \left.\times \sin \left[2 \pi\left(u_{2} x+v_{2} y\right)\right]\right\} \\
= & k_{2} c^{2}\left\{\cos \left[2 \pi\left\{\left(u_{1}-u_{2}\right) x+\left(v_{1}-v_{2}\right) y\right\}\right]\right. \\
& \left.-\cos \left[2 \pi\left\{\left(u_{1}+u_{2}\right) x+\left(v_{1}+v_{2}\right) y\right\}\right]\right\} .(3)
\end{aligned}
$$

The two new components introduced by the quadratic nonlinearity have spatial frequencies and orientations determined by the characteristics of the original gratings. (Terms contributed by cubic and higher order powers in the polynomial contribute distortion products of still different orientations and spatial frequencies.) The contrast of the distortion products relative to those of the generating stimuli depend on the form of the nonlinearity which in turn determines the value of $k_{i}$, the coefficient of the $i$ th term in the power-series expansion of the nonlinearity. When the contrast is small, higher order terms of likely non-linearities produce distortion products with negligible contrast.

Now for our symmetrically orientated twocomponent masker, $u_{i}$ and $v_{i}$ are the functions of spatial frequency and orientation given by,

$$
\begin{aligned}
& u_{1}=f \cdot \cos (2 \pi \theta / 360), \\
& v_{1}=f \cdot \sin (2 \pi \theta / 360),
\end{aligned}
$$

and

$$
\begin{aligned}
& u_{2}=-f \cdot \cos (2 \pi \theta / 360), \\
& v_{2}=f \cdot \sin (2 \pi \theta / 360),
\end{aligned}
$$

where $f$ is the spatial frequency of the masker and $\theta$ is the magnitude of its angle (in degrees) relative to that of the signal (Gaskill, 1978).
When equation 4 is substituted into equation 3 , the quadratic distortion product becomes,

$$
\begin{array}{r}
D_{2}(x, y)=k_{2} c^{2}\{\cos [2 \pi 2 f x \cdot \cos (2 \pi \theta / 360)] \\
-\cos [2 \pi 2 f y \cdot \sin (2 \pi \theta / 360)]\} .
\end{array}
$$

The components of equation 5 have spatial frequencies that are $2 \cdot \cos (2 \pi \theta / 360)$ and $2 \cdot \sin (2 \pi \theta / 360)$ times that of the generating gratings; one is horizontal and the other vertical. And it might be expected that the vertically orientated distortion product produces the masking we measure.

\section{GENERAL DISCUSSION}

That there should be masking with gratings 45 and $67.5 \mathrm{deg}$ from the signal orientation is not inconsistent with the notion of their being a nonlinear component generated in response to the sum of the two masking gratings; in Expt I, there is a large masking effect. If we accept that explanation, then the fact that the nonlinear interaction occurs between components of such different orientation suggests a possible site of interaction prior to orientation tuning; that is, in or prior to the lateral geniculate nucleus where distortion products from identically orientated gratings have recently been demonstrated (Derrington, 1987a).

However one important feature of Figs 3 and 4 that is not consistent with the notion that the masking is produced by a pre-striate nonlinearity is the relation between threshold elevation and spatial frequency. The maximum masking effect is very close to the spatial frequency of the components of the masker and not near the spatial frequency of the distortion product which, in the 22.5 and 45 deg cases is well above the masker frequency. Nor does the location of the maximum decrease as the cosine of the relative orientation of the signal and the maskers in the way it should if the masking were being produced by a (quadratic) distortion product. Moreover, the masking functions are similar in shape to those obtained with noise maskers; they lack the sharp peak at the masker frequency characteristic of narrow-band maskers (Quick and Reichert, 1975; Henning and Hinton, unpublished data); however one interprets the presence of such a peak, its absence clearly indicates the absence of a significant sinusoidal distortion product. Finally, and in addition, masking with orientation-limited noise at the three orientations shows only nondecreasing functions of the masking noise con- 
trast (Henning, 1988b); at these orientations no "pedestal effect" is seen. The usual inference is that the masker and signal are travelling in separate channels. But, if they are in separate channels, there should be no interaction which there undoubtedly is (Figs 3 and 4). Thus the notion of a peripheral (LGN) nonlinearity as the source of the masking breaks down. Nor does the notion of orientation-tuned channels receive support unless one assumes that postchannel combination of information produces the masking. This is not an unreasonable assumption but clearly illustrates the futility of attempting to explain even the relatively simple visual discrimination results we present with a model based on linear mechanisms tuned for spatial frequency and orientation.

\section{SUMMARY}

(1) Pairs of gratings that are symmetrically orientated on either side of a vertical signal grating produce large masking effects.

(2) The effects depend very little on orientation.

(3) In this respect alone the result is consistent with the presence of pre-cortical quadratic nonlinearity operating on luminance.

(4) We fail to find either sharply tuned spatial-frequency masking functions or even secondary peaks near the spatial frequency of a major distortion product; these findings make nonlinear distortion products an unlikely cause of the masking we measure.

(5) Even simple discrimination results are inconsistent with the notion that simple visual discriminations are significantly limited by the orientation-tuned mechanisms of early cortical processing.

Acknowledgement-A.M.D. received a travel grant from the Guarantors of Brain.

\section{REFERENCES}

Blakemore C. and Campbell F. W. (1969) On the existence of neurones in the human visual system selectively sensitive to the orientation and size of retinal images. J. Physiol. Lond. 203, 237-260.

Burbeck C. A. and Kelly D. H. (1981) Contrast gain measurements and the transient/sustained dichotomy. J. opt. Soc. Am. 71, 1335-1342.

Campbell F. W. and Robson J. G. (1968) Application of
Fourier analysis to the visibility of gratings. $J$. Physiol. Lond. 197, 551-566.

Carlson C. R. and Klopfenstein R. W. (1985) Spatialfrequency model for hyperacuity. J. opt. Soc. Am. A 2 , 1747-1751.

Carter B. E. and Henning G. B. (1971) The detection of gratings in narrow-band visual noise. J. Physiol. Lond. 219, 355-365.

Derrington A. M. (1987a) Distortion products in geniculate $X$-cells: a physiological basis for masking by spatially modulated gratings? Vision Res. 27, 1377-1386.

Derrington A. M. (1987b) Amplitude-modulated gratings mask the response of $X$-cells in cat LGN to gratings of the modulation frequency. J. Physiol. Lond. 391, 39P.

De Valois R. L., Albrecht D. G. and Thorell L. (1982) Spatial frequency selectivity of cells in macaque visual cortex. Vision Res. 22, 545-559.

Ferrara V. P. and Wilson H. R. (1987) Direction specific masking and the analysis of motion in two dimensions. Vision Res. 27, 1783-1796.

Gaskill J. D. (1978) Linear Systems, Fourier Transforms and Optics. Wiley, New York.

Gelinski A. (1968) Orientation-specific effects of patterns of adapting light on visual acuity. J. opt. Soc. Am. 58, 13-18.

Greis U. and Roehler R. (1970) Untersuchungen der subjectiven Detail-Erkenbarkeit mit Hilfe der Ortsfrequenzfilterung. Optica Acta 17, 516-526.

Henning G. B. (1988) Spatial-frequency tuning as a function of temporal frequency and stimulus motion. $J$. opt. Soc. Am. A 5, 1362-1373.

Henning G. B. and Hinton J. L. (1988) Unpublished data.

Henning G. B., Hertz B. G. and Broadbent D. E. (1975) Some experiments bearing on the hypothesis that the visual system analyses spatial patterns in independent bands of spatial frequency. Vision Res. 15, 887-897.

Henning G. B., Hertz B. G. and Hinton J. L. (1981) Effects of different hypothetical detection mechanisms on the shape of spatial-frequency filters inferred from masking experiments. J. opt. Soc. Am. 71, 574-581.

Legge G. E. and Foley J. M. (1980) Contrast masking in human vision. J. opt. Soc. Am. 70, 1458-1471.

Maffei L. and Fiorentini A. (1973) The visual cortex as a spatial frequency analyser. Vision Res. 13, 1255-1268.

Nachmias J. and Rogowitz B. E. (1983) Masking by spatially-modulated gratings. Vision Res. 23, 1621-1629.

Pantle A. and Sekuler R. (1968) Size-detecting mechanisms in human vision. Science 162, 1146-1148.

Phillips E. C. and Wilson H. R. (1984) Orientation bandwidths of spatial mechanisms measured by masking. J. opt. Soc. Am. A 1, 226-232.

Quick R. F. and Reichart T. A. (1975) Spatial frequency selectivity in contrast detection. Vision Res. 15, 637-643.

Rabiner L. R. and Gold B. (1975) Theory and Applications of Digital Signal Processing. Prentice-Hall, Englewood Cliffs.

Regan M. P. and Regan D. (1987) Orientation tuning and spatial frequency tuning of a phase-independent brain response. Invest. Ophthal. visual Sci. 28, S127 [A].

Schade O. H. (1956) Optical and photoelectric analog of the eye. J. opt. Soc. Am. 46, 721-739.

Stromeyer C. H. and Julesz B. (1972) Spatial frequency masking in vision: critical bands and the spread of masking. J. opt. Soc. Am. 62, 1221-1232. 